\title{
Emprendimiento Social en los Estudiantes del Instituto Tecnológico de Cd. Cuauhtémoc
}

\section{Social Entrepreneurship in the Students of the Technological Institute of Cd. Cuauhtémoc}

\author{
Ema Cristina Gutiérrez Enríquez*, Karla Isabel Gutiérrez Enríquez**, \\ Alejandra Jaques Sandoval***, Grecia Sandoval Acosta***,

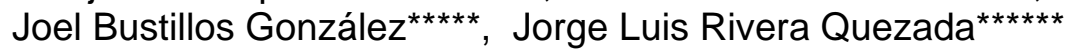

*Maestra en Ciencias en Psicología Social de las Organizaciones. Tecnológico Nacional de México. ORCID: http://orcid.org/0000-0001-8639-7085.

**Ingeniera Industrial. Instituto Tecnológico de Chihuahua, Departamento de Ingeniería Industrial. Cuauhtémoc, Chihuahua. ORCID: http://orcid.org/0000-0002-7515-144X.

***Estudiante de la Licenciatura en Administración. Instituto Tecnológico de Ciudad Cuauhtémoc. Cuauhtémoc, Chihuahua. ORCID: http://orcid.org/0000-0002-4493-8741.

****Estudiante de la Licenciatura en Administración. Instituto Tecnológico de Ciudad Cuauhtémoc. Cuauhtémoc, Chihuahua. ORCID: http://orcid.org/0000-0003-0732-4086.

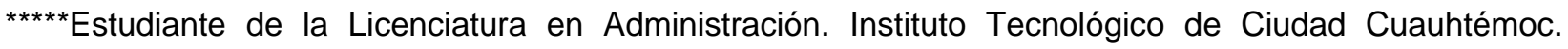
Cuauhtémoc, Chihuahua. ORCID: http://orcid.org/0000-0002-7493-2447.

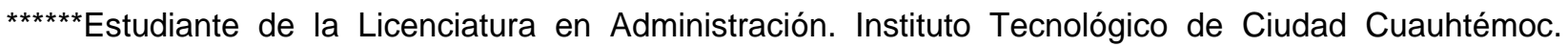
Cuauhtémoc, Chihuahua. ORCID: http://orcid.org/0000-0002-2714-8772.

Correo para recibir correspondencia: cristigutie@gmail.com

Fecha de recibido: 3 de noviembre de 2020

Fecha de aceptación: 11 de diciembre de 2020

GUTIÉRREZ-ENRÍQUEZ E C. GUTIÉRREZ-ENRÍQUEZ K. I., JAQUES-SANDOVAL A., SANDOVAL-ACOSTA G., BUSTILLOSGONZÁLEZ J., RIVERA-QUEZADA J. L. 
EMPRENDIMIENTO SOCIAL EN LOS ESTUDIANTES DEL INSTITUTO TECNOLÓGICO DE CD. CUAUHTÉMOC.

\section{RESUMEN}

OBJETIVO: Conocer las características de emprendimiento y conciencia social de los estudiantes del Instituto Tecnológico de Cd. Cuauhtémoc.

MATERIAL Y MÉTODO: Se aplicó una escala tipo Likert de 25 preguntas, a 200 estudiantes del Instituto Tecnológico de Cd. Cuauhtémoc, todos ellos de diferentes carreras y semestres.

RESULTADOS: Se encontró que los niveles de emprendimiento y conciencia social son bajos, contrario a lo esperado.

CONCLUSIONES: Se observa muy poca tendencia al emprendimiento, se observa conformismo y el nivel de conciencia social también es bajo, no hay apoyo comunitario.

PALABRAS CLAVE: Emprendimiento. Emprendimiento social. Conciencia social.

\section{ABSTRACT}

OBJECTIVE: To know the characteristics of entrepreneurship and social awareness in the students of the Technological Institute of Cd. Cuauhtémoc.

MATERIAL AND METHOD: A Likert-type scale of 25 questions was applied to 200 students from the Technological Institute of $\mathrm{Cd}$. Cuauhtémoc, all of them from different careers and semesters.

RESULTS: It was found that the levels of entrepreneurship and social awareness are low, contrary to expectations.

CONCLUSIONS: Very little tendency to entrepreneurship is observed, conformity is observed and the level of social awareness is also low, there is no community support.

KEY WORDS: Entrepreneurship, social entrepreneurship and social awareness. 
EMPRENDIMIENTO SOCIAL EN LOS ESTUDIANTES DEL INSTITUTO TECNOLÓGICO DE CD. CUAUHTÉMOC.

\section{INTRODUCCIÓN}

El concepto de emprendedor social comienza a tomar auge en los años 80's según el Instituto Nacional de Economía Social (2018), cuando la persona se enfoca a resolver problemáticas sociales y mediante su proyecto de negocio promueve la solución de dichos problemas. Un emprendedor tiene la capacidad de detectar y aprovechar las oportunidades de negocio, en este caso, su enfoque es social, destinando los recursos organizacionales al beneficio de la comunidad.

La Fundación Seres (2017), considera que los emprendedores sociales tienen la capacidad de promover cambios con impacto en la sociedad, así mismo proponen que hay tres ejes en el emprendimiento social, en primer lugar, deben ser el motor de cambio, ayudando a mejorar y desarrollar la vida de quién lo requiere y no ha sabido como hacerlo. En segundo término, se debe buscar la oportunidad de generar valor social con el cambio; es decir, plantear objetivos positivos y promover las herramientas e insumos necesarios para alcanzarlos. Por último, que ese deseo de mejorar y apoyar a los demás se ramifique, se contagie la energía e ideología del emprendimiento.

A través de los tiempos, la humanidad ha mostrado tener emprendimiento social, existen casos muy impactantes como Steve Jobs quién proporciono al mundo una forma revolucionaria de procesar la información mediante el ordenador y revoluciono la comunicación mediante el teléfono móvil. Existen fundaciones que dan microcréditos a la ciudadanía para que desarrollen microempresas, las cuales generan crecimiento económico en su localidad, algunas fundaciones instalan clínicas u hospitales médicos que promueven la salud en comunidades rurales. También existen pensadores enfocados al emprendimiento social, mediante la ideología de derechos humanos, tal es el caso de Martin Luther King, Mahatma Gandi, entre otros.

Ivarbo, Quijano y Portillo (2018), mencionan que "el emprendimiento social busca crear un tipo de empresa que va dirigida a resolver problemas sociales o ambientales como lo son el desempleo, racismo, exclusión social, obesidad, tala excesiva, entre otros", para los autores las características del emprendedor social son el tener un gran compromiso con la causa, enfrentan y resisten las situaciones difíciles, corren riesgos, les preocupa la marginación, la exclusión, son generosos y aceptan la innovación, disfrutan de la vida y practican acciones de voluntariado. Los autores citan a Van Ryzin, Grossman, DiPadova-Stocks \& Bergrud (2009), quienes argumentan GUTIÉRREZ-ENRÍQUEZ E C. GUTIÉRREZ-ENRÍQUEZ K. I., JAQUES-SANDOVAL A., SANDOVAL-ACOSTA G., BUSTILLOSGONZÁLEZ J., RIVERA-QUEZADA J. L. 
EMPRENDIMIENTO SOCIAL EN LOS ESTUDIANTES DEL INSTITUTO TECNOLÓGICO DE CD. CUAUHTÉMOC.

que las personas jóvenes tienen mayor disposición para el emprendimiento social, así como citan a Harding (2006), que reconoce que la experiencia laboral y el alto grado académico facilitan la práctica del emprendimiento social. No hay una diferencia marcada entre hombres y mujeres, los hombres son más emprendedores y las mujeres también tienden a realizar los proyectos de beneficio social.

\section{MARCO TEÓRICO CONCEPTUAL}

Según Neetwork business school (2020), el emprendimiento social surge en los años sesenta y setenta, cuando se empieza a hablar del cambio social, lo que es bastante actual es el clasificar las "empresas socialmente emprendedoras como Empresas Sociales", ya que están dispuestas a financiar de manera económica las iniciativas de apoyo a la comunidad. Los autores consideran que:

El emprendimiento social, por su naturaleza, está esencialmente limitado por la misión social y la teoría del cambio. La percepción general de equiparar el emprendimiento social con la creación de organizaciones sin fines de lucro, probablemente surge de las fuertes misiones sociales que persiguen estas organizaciones.

Nuño (2017), menciona que la evolución social está siendo afectada por varios factores tanto endógenos como exógenos, todo ello lleva al establecimiento de objetivos y estrategias empresariales distintos a los comunes. Actualmente surgen empresas con carácter de beneficio, enfocadas al emprendimiento social, que se entiende como las empresas preocupadas por el crecimiento social, el cuidado del medio ambiente, sin fines de lucro y conciencia social. Respecto a esto Carranza (2020), menciona que "el emprendimiento social es la fusión entre una solución innovadora a un problema de desarrollo socio-económico y una estrategia sostenible con impacto medido". El autor menciona que la Fundación Ashoka "define un emprendedor social como una persona con soluciones innovadoras a los problemas más graves en sus comunidades". También considera que los emprendedores sociales son ambiciosos y persistentes, enfrentando los problemas de desarrollo social, cultural y económico en una comunidad. El emprendedor social no se atiene a que el gobierno o el sector privado traten de solucionar un problema social, se responsabiliza y enfrente activamente la situación. 
EMPRENDIMIENTO SOCIAL EN LOS ESTUDIANTES DEL INSTITUTO TECNOLÓGICO DE CD. CUAUHTÉMOC.

Para Veredith (2020), argumenta que "el emprendimiento social consiste en la satisfacción de una necesidad social o medio ambiental por medio de una institución que genera beneficios económicos y se re-invierte en conseguir el objetivo social principal", así mismo se observa el emprendimiento social en las organizaciones sin fines de lucro, ya que estas no trabajan por un beneficio económico y tienen beneficios sociales. Hoy en día el emprendimiento social está de moda, es el objeto de interés y atención de la población, ya que tiene la capacidad de solventar y afrontar problemas sociales en los momentos y situaciones en los que el sector público, el mercado y las organizaciones no gubernamentales no pueden hacerlo.

El emprendimiento social implica para Raffino (2018), emprender con el objetivo de generar un beneficio social, ecológico y de apoyo comunitario, es decir, crear empresa sin fines de lucro, con conciencia social. Esta forma de emprendimiento plantea objetivos y metas con impacto comunitario, local, estatal, nacional o internacional, mediante el financiamiento gratuito de proyectos. Dentro de este tipo de organizaciones se encuentran las cooperativas, Organizaciones no gubernamentales (ONG's), fundaciones, que trabajan con recursos gubernamentales o mediante asociaciones civiles.

Para Carazo-Alcalde (2020), el emprendimiento social es "la puesta en marcha de una empresa cuyo objetivo final no es la maximización del beneficio económico, sino la creación de valor para la sociedad". El objetivo de este emprendimiento está enfocado a satisfacer necesidades sociales y del medio ambiente, invierten su capital en fines sociales. Para considerar una organización con emprendimientos social, es necesario contener varios elementos, entre ellos tener como objetivo el cambio social generando valor o crecimiento social, se debe proponer soluciones innovadoras a los problemas cotidianos (pobreza, hambruna, mala salud, exclusión social, etc.), la propia empresa es el medio para alcanzar los objetivos, sus ganancias económicas se reinvierten en el mismo beneficio social. La autora considera que no se debe confundir el emprendimiento social con la innovación social, el emprendimiento social en la empresa puede ser una innovación social.

Disruptivo.tv (2019), define el emprendimiento social como "empresas enfocadas en resolver los problemas sociales con un modelo de negocio que le permite ser escalable, sustentable y generar utilidades". Buscan minimizar las necesidades sociales mediante la aplicación de estrategias de negocio entre ellas la inversión, ventas, producción. Esto surge de la fusión de una empresa sin

GUTIÉRREZ-ENRÍQUEZ E C. GUTIÉRREZ-ENRÍQUEZ K. I., JAQUES-SANDOVAL A., SANDOVAL-ACOSTA G., BUSTILLOSGONZÁLEZ J., RIVERA-QUEZADA J. L. 
EMPRENDIMIENTO SOCIAL EN LOS ESTUDIANTES DEL INSTITUTO TECNOLÓGICO DE CD. CUAUHTÉMOC.

fines de lucro y la empresa tradicional que se rige por modelos sustentables. Así mismo el Instituto Nacional de Economía Social (2018), menciona, que el emprendimiento social se ha ido extendiendo cada día, en las universidades se ofrecen asignaturas enfocadas a este objetivo, así como el gobierno ofrece apoyos económicos para lograr cambiar las formas de emprendes, trabajo y producción. Esta novedosa forma de trabajo, se aplica en cualquier rama de producción, buscando el bienestar social y mejorar las formas y procesos.

Raffino (2018), comenta que las organizaciones con enfoque de emprendimiento social buscan el triple resultado, impactando en lo financiero, social y medioambiente. Por tanto, surgen diferentes tipos o formas del emprendimiento social, entre ellas está el de promoción que se encarga de difundir y promover sus causas, el de acción que se enfoca de resolver problemas comunitarios, el de especialistas reúne profesionales de diferentes enfoques que se unen con un interés social. La forma de financiamiento varia puede ser privada o gubernamental, o autogestión financiera. Características del emprendimiento social según el autor:

a) Un emprendimiento social se desarrolla sin fines de lucro.

b) Tiene como objetivo la solución o disminución de los problemas de tipo comunitario, social o medioambiental, con la finalidad de mejorar la vida humana.

c) Emplea estrategias y métodos empleados de forma comercial o empresarial, como la administración, publicidad, organización, etc.

d) Proporciona empleo a sus colaboradores de la misma forma que lo hacen las demás organizaciones.

Nuño (2017), expresa que la sociedad se empeña cada día por mejorar las condiciones ambientales y sociales, lo que lleva a las organizaciones a actuar con ética, incluso los consumidores son cada vez más conscientes de elegir los productos biodegradables y con menor daño al ambiente, las exigencias del mercado son cada vez mayores, por lo que las empresas deben adaptarse a esta nueva forma de consumo. Las tendencias de mercado van cada día encaminadas a un cambio social y económico, donde se manifieste la conciencia social de apoyo, esto se observa en los programas de redondeo o de donación para una fundación, en la fabricación y utilización de productos biodegradables o amigables con el medio ambiente.

Neetwork business school (2020), proponen cuatro pilares de las empresas sociales:

GUTIÉRREZ-ENRÍQUEZ E C. GUTIÉRREZ-ENRÍQUEZ K. I., JAQUES-SANDOVAL A., SANDOVAL-ACOSTA G., BUSTILLOSGONZÁLEZ J., RIVERA-QUEZADA J. L. 
EMPRENDIMIENTO SOCIAL EN LOS ESTUDIANTES DEL INSTITUTO TECNOLÓGICO DE CD. CUAUHTÉMOC.

a) La viabilidad económica del proyecto: Los proyectos de emprendimiento social deben encaminarse a cubrir una necesidad comunitaria, previamente detectada en un estudio de mercado, al igual que una actividad empresarial, por lo que es indispensable que produzca una ganancia y genere empleos.

b) Objetivos sociales y / o ambientales: Los proyectos de emprendimiento social deben contar con metas y objetivos encaminados a la mejora social y/o ambiental, pudiendo enfocarse a mejoras de salud, energías renovables, actividades económicas como el empleo, producción de alimentos naturales, fomento y creación de empresas locales.

c) Distribución de ganancias: Algunas empresas reinvierten sus ganancias en el logro de sus objetivos sociales y de mejoramiento del medio ambiente.

d) Gobernación: "Participación es la palabra clave de gobernanza en el emprendimiento social". Se deben conciliar los intereses colectivos y los intereses individuales, la toma de decisiones es responsabilidad del grupo, desde los gerentes, personal de la empresa, los clientes y las instancias de gobierno.

La acción de emprender generalmente surge por la idea de hacer algo que satisfaga las necesidades de la población, en los tiempos de crisis, se reflexiona y surgen propuestas de cambio que beneficien a la comunidad, según Emprendimiento social (2020), varios intentos de emprendimiento no llegan a concretarse, debido a innumerables factores; sin embargo, en los tiempos de dificultad social, las ideas deben ser más sólidas para poder llevarse a acabo. Ser emprendedor no es sólo iniciar con la creación de una empresa, debe ser todo un estilo de vida, una de las motivaciones del emprendedor es ser su propio jefe. Los autores citan textualmente que "cuando la idea de emprender se apodera de alguien, lo empieza a rodear una energía única que lo llena de entusiasmo y que le da las ganas para querer concretar un concepto que será parte de su vida".

Semana Educación (2017) entrevistó a Jaime Alberto Rincón, rector de la Corporación Unificada Nacional para saber de qué se trata, "el emprendimiento social es la fusión entre una solución innovadora a un problema de desarrollo social y económico y una estrategia sostenible de impacto". Para ello es importante la capacitación de los profesionistas en este aspecto de tener conciencia social, el emprendimiento social es de suma importancia debido a la crisis económica mundial y a la falta de valores sociales que se presenta actualmente, por ello algunas empresas han dado un giro a sus enfoques y se han dedicado a solucionar problemas de tipo social en las GUTIÉRREZ-ENRÍQUEZ E C. GUTIÉRREZ-ENRÍQUEZ K. I., JAQUES-SANDOVAL A., SANDOVAL-ACOSTA G., BUSTILLOSGONZÁLEZ J., RIVERA-QUEZADA J. L. 
EMPRENDIMIENTO SOCIAL EN LOS ESTUDIANTES DEL INSTITUTO TECNOLÓGICO DE CD. CUAUHTÉMOC.

comunidades, buscando el sostenimiento de ellas mismas, mediante el apoyo que brindan. El concepto de "innovación se ha convertido en una moda los últimos años, pero siempre ha existido como herramienta de construcción. La innovación se puede dar no solo desde la tecnología, sino también desde cualquier ámbito organizacional".

Neetwork business school (2020), menciona que se podría decir que los "emprendedores sociales son como albañiles que vienen a remendar los agujeros que una comunidad padece, ya sea por impericia, falta de recursos o desconocimiento del Estado responsable". Los considera personas en forma individual o grupos de individuos que perciben una carencia social, un problema o una oportunidad de cambio e intentan darle una solución o mejorar las condiciones. Los emprendedores sociales crean de forma novedosa soluciones a las condiciones sociales que requieran un cambio, así mismo asumen riesgos y se esfuerzan en lograrlo. Estos autores argumentan que en el compromiso social, "el uso generalizado de prácticas éticas como la inversión de impacto, el consumo consciente y los programas de responsabilidad social corporativa facilitan el éxito de los emprendedores sociales".

UnLtd Spain (2019), considera que los emprendedores sociales son personas que se enfocan en iniciar y desarrollar una empresa o proyecto con enfoque de beneficio social, ellos tienen varias características, como la confianza en sí mismo, ya que al conocerse a sí mismo, sabrá cuáles son sus habilidades, así como fortalezas y debilidades, buscan trabajar en equipo para fortalecer sus alcances, otra de sus características es la fuerte motivación que los impulsa a trabajar duro con una visión real del futuro, las capacidades y el logro. Asimismo, tienen capacidad de planificar y organizar y pueden mantener la comunicación de manera eficaz. Otras habilidades que poseen los emprendedores sociales son la pasión por sus proyectos, liderazgo, son innovadores, bastante resilientes, pueden tomar decisiones en momentos difíciles, son inconformes con los problemas sociales, gran responsabilidad y compromiso social; es decir, hablamos de aquellas personas que no se conforman con lo preestablecido porque se cuestionan la realidad o porque tienen historias personales detrás que les motivan a emprender. Buscan un compromiso con aquello que no les gusta de la sociedad, haciendo su trabajo con pasión, pero también con responsabilidad. Ofrecer soluciones a los problemas sociales no siempre es fácil. Por eso, la adaptación al contexto y la coherencia entre las actividades y el propósito de la empresa son siempre fundamentales para entender la mente de un emprendedor social y la continuidad de su proyecto.

GUTIÉRREZ-ENRÍQUEZ E C. GUTIÉRREZ-ENRÍQUEZ K. I., JAQUES-SANDOVAL A., SANDOVAL-ACOSTA G., BUSTILLOSGONZÁLEZ J., RIVERA-QUEZADA J. L. 
EMPRENDIMIENTO SOCIAL EN LOS ESTUDIANTES DEL INSTITUTO TECNOLÓGICO DE CD. CUAUHTÉMOC.

Los autores concluyen que no hay una definición exacta de emprendedor social y tampoco se han definido las características propias y comunes a todos ellos. Lo que si queda muy claro es que se trata de personas que trabajan como agentes de cambio que encabezan los proyectos que dan solución a los grandes desafíos, demostrando así que se puede emprender en pro de los problemas de la comunidad.

Rankia (2019), argumentan que los emprendedores sociales son individuos que buscan establecer cambios en la sociedad mediante el liderazgo, visión, innovación y creatividad para minimizar y solucionar algunos problemas que perjudican a los grupos sociales, sobre todo en los sectores más vulnerables como son la educación, el medio ambiente, la sociedad, la salud, pobreza y la discriminación. Los autores mencionan textualmente que:

Actualmente, en la sociedad se encuentra un grupo de personas que rompen con los esquemas y patrones, ya que centran sus conocimientos y capacidades en asumir riesgos que les permitan lograr proyectos visionarios enfocados en modificar, mejorar y encontrar alternativas para resolver una problemática social que transforme el modo de vida de la gente. A esta persona se le conoce como un emprendedor social quien es capaz de aportar sus ideas para mejorar e innovar un sector de la sociedad.

Las aptitudes que debe poseer el emprendedor social según los autores son el tener visión de negocios con enfoque de resolver problemas sociales, deberán ser perseverantes, constantes, saber trabajar en equipo, poder de convencimiento hacia el cambio, liderazgo, capacidad innovadora, creatividad, planear estratégicamente, compromiso y responsabilidad social; así como, mostrar pasión, asumir riesgos sin esperar un beneficio económico a cambio. Los emprendedores sociales deberán estar conscientes que su labor es altruista, ya que son cada vez más los ciudadanos que requieren apoyo y mayores los problemas comunitarios que necesitan atención.

Carazo-Alcalde (2020), considera que un emprendedor social debe caracterizarse por su conciencia social, detectando problemas y querer participar en resolverlos, deberá tener capacidad de emprender y liderar, esto conlleva a buscar financiamiento para la elaboración de su producto o el servicio que va a brindar. Del Cerro (2015), considera que el emprendimiento social es una nueva forma de ver y hacer negocio, las generaciones de ciudadanos jóvenes van teniendo mayor conciencia social, es probablemente debido a la problemática social y ambiental GUTIÉRREZ-ENRÍQUEZ E C. GUTIÉRREZ-ENRÍQUEZ K. I., JAQUES-SANDOVAL A., SANDOVAL-ACOSTA G., BUSTILLOSGONZÁLEZ J., RIVERA-QUEZADA J. L. 
EMPRENDIMIENTO SOCIAL EN LOS ESTUDIANTES DEL INSTITUTO TECNOLÓGICO DE CD. CUAUHTÉMOC.

que se experimenta en la actualidad, en México se ha observado el crecimiento en el número de empresas que buscan la mejora social y ambiental. El autor recomienda a los empresarios que exploren las páginas oficiales en México, especializadas en emprendimiento social, entre ellas están el Instituto Nacional del Emprendedor (INADEM) y la Secretaría de Innovación, Ciencia y Tecnología (SICyT), así como asistir a los eventos dedicados a este rubro.

Fuentes y cols., (2018), exponen que el CONEVAL considera:

Que el 46\% de los mexicanos viven en alguna situación de pobreza y el 10.4\% se encuentra en pobreza extrema, esto nos lleva a pensar y crear emprendimientos con el objetivo de solucionar ciertos problemas que los empresarios en décadas no han podido hacer frente y cortar los problemas de raíz.

Las malas condiciones económicas en México y la falta de oportunidades de empleo, ha obligado a la población a considerar el trabajo en equipo para salir adelante como sociedad, esta molestia social lleva a buscar el cambio con desesperación, impaciencia y esfuerzo, pero se aconseja tomar las cosas con calma y planear detenidamente para no poner el proyecto en riesgo y a sus colaboradores. En cuestión de financiar los proyectos, es recomendable buscar financiadoras de microcréditos, que están hechos a la necesidad de las microempresas.

\section{MATERIAL Y MÉTODO}

El presente estudio se llevó a cabo en el Instituto Tecnológico de Cd. Cuauhtémoc, mediante la aplicación del instrumento de medición de forma personal con cada uno de los alumnos participante. Es de tipo cuantitativo y descriptivo, ya que muestra la tendencia de los alumnos a participar en acciones de emprendimiento y conciencia social. El análisis de los datos es mediante estadística descriptiva.

Muestra: La muestra es de tipo conveniencia, ya que se aplicó la encuesta a 200 estudiantes del Instituto Tecnológico de Cd. Cuauhtémoc, todos ellos con la disponibilidad de brindar información, los participantes fueron de todos los semestres y de todas las carreras. Se consideraron las variables sociodemográficas sexo genérico, semestre y carrera de los encuestados, para efectos del análisis de los datos. 
EMPRENDIMIENTO SOCIAL EN LOS ESTUDIANTES DEL INSTITUTO TECNOLÓGICO DE CD. CUAUHTÉMOC.

Instrumento: Se obtuvieron los datos mediante la aplicación de una escala tipo Likert de 25 preguntas (ítems), desarrollado por Ivarbo, Quijano y Portillo (2018), que detecta el actuar de los individuos para definir el nivel de emprendimiento y de conciencia social, la escala tiene un índice de confiabilidad Alfa de Cronbach de .80 , además hay 8 preguntas para datos sociodemográfico.

\section{RESULTADOS}

Con el presente estudio se busca detectar comportamientos en los individuo que favorecen el Emprendimiento social (ver figura 1), tal como: la capacidad de manejar la realidad social y conciencia del otro, se observa a través del manejo de los valores como la tolerancia, la empatía, liderazgo, aceptar las diferencias culturales y religiosas; otro comportamiento es la benevolencia, con ella se busca el bienestar de las personas por medio de la interacción diaria con ellos, amistad, lealtad; aquí los valores específicos de conformidad son obediencia y buenos modales; también se pretende detectar comportamientos de conciencia social (ver figura 2), como son: la sensibilidad social, con ella se muestran conductas de rechazo a la exclusión, marginación y aceptación de las necesidades de grupo; y por último, está la credibilidad para terceros, el manejo de un código ético observando credibilidad, responsabilidad y coherencia al actuar y pensar.

Los resultados obtenidos con el análisis de los datos, son los siguientes:

\section{Emprendimiento social:}

El 28.5\% de los encuestados manifiesta que prefiere ser el líder de su equipo al realizar trabajos escolares, al 9.5\% de ellos le gusta proponer el lugar de reunión; el 27\% ayuda a sus compañeros a realizar el trabajo y estudiar; el 30\% se esfuerza para desarrollar sus proyectos nuevos, el $14.5 \%$ busca diferentes alternativas para realizar su proyecto, mientras el $10.5 \%$ solo se enfoca en realizar solo lo indispensable; el $24.5 \%$ hace un esfuerzo extra en sus tareas escolares y el $9 \%$ no lo hace; el 19\% se asegura de tener los conocimientos necesarios para realizar sus tareas; el $58.5 \%$ de los estudiantes se arriesga a ir a otra ciudad a estudia; el $47 \%$ de ellos manifiesta que si se arriesga a tomar materias de su carrera que son difíciles; el 35\% expone que reconocen ser responsables cuando faltan a clase, y el 23\% manifiesta que copiaría en un examen de tener la oportunidad mientras que el $12 \%$ dice que no lo haría. 
EMPRENDIMIENTO SOCIAL EN LOS ESTUDIANTES DEL INSTITUTO TECNOLÓGICO DE CD. CUAUHTÉMOC.

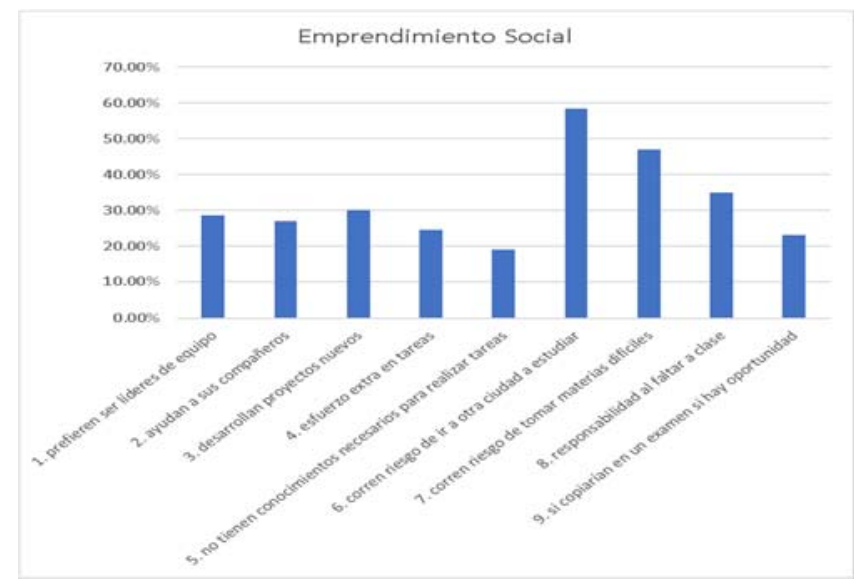

Figura 1. Comportamientos de emprendimiento social.

Fuente: Elaboración propia.

2. Conciencia social:

El 18.5 de los encuestados se involucra en problemas comunitarios y trata de ser parte de la solución el 8\% evita involucrarse; el 8.5\% considera que debe apoyar para mejoras de su colonia mientras el $12.5 \%$ piensa que no debe apoyar; el 23.5\% apoya programas de beneficencia social., el $26 \%$ expresa que no apoya a los indigentes, el $9.5 \%$ si lo hace; el $12 \%$ piensa que debe de ayudar a los pobres mientras el $6 \%$ piensa que no debe ayudarlos, ya que son pobres porque no se esfuerzan; el $12.5 \%$ piensa que los adultos mayores deben participar en actividades productivas y el $12.5 \%$ cree que ya no deben hacerlo; el 58\% nunca ha donado sangre y el $6 \%$ si lo ha hecho; el 35\% no participa en las actividades de la iglesia y el $8 \%$ si lo hace.

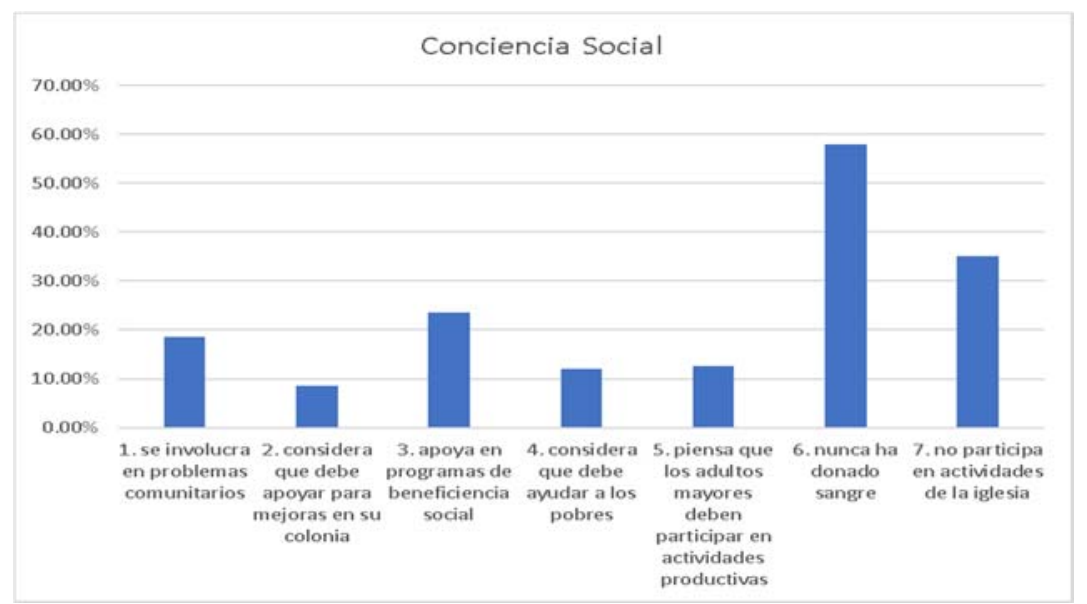

Figura 2. Comportamientos de conciencia social.

Fuente: Elaboración propia.

GUTIÉRREZ-ENRÍQUEZ E C. GUTIÉRREZ-ENRÍQUEZ K. I., JAQUES-SANDOVAL A., SANDOVAL-ACOSTA G., BUSTILLOSGONZÁLEZ J., RIVERA-QUEZADA J. L. 
EMPRENDIMIENTO SOCIAL EN LOS ESTUDIANTES DEL INSTITUTO TECNOLÓGICO DE CD. CUAUHTÉMOC.

3. Resultados generales:

Se analizaron los datos de las variables sociodemográficas, encontrando lo siguiente: De los 200 alumnos encuestados, 110 son del sexo masculino que representan el 55\% y 90 son del sexo femenino que representan el $45 \%$.

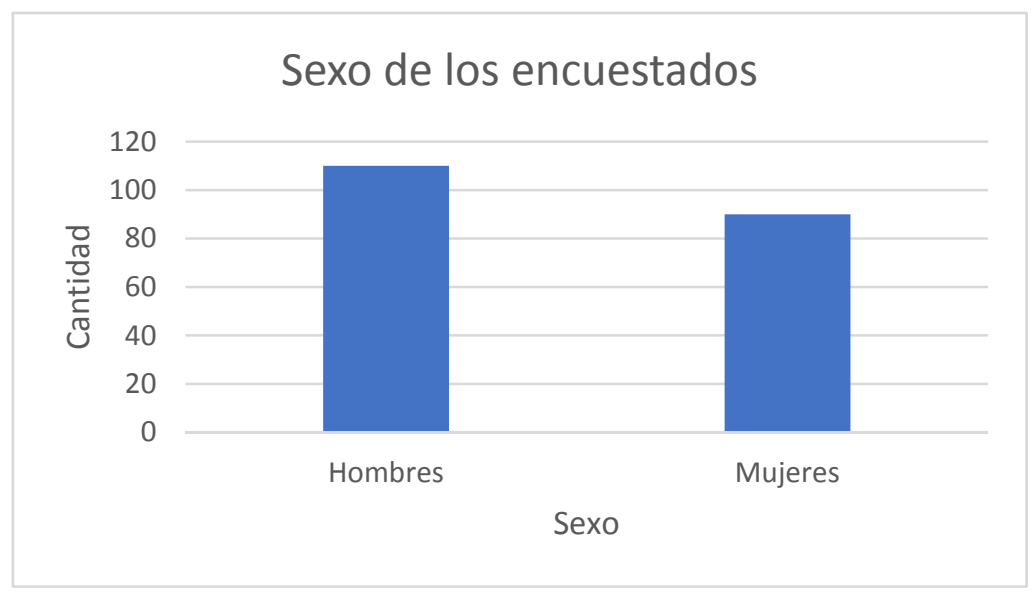

Figura 3. Porcentaje de alumnos por sexo genérico.

Fuente: Elaboración propia.

De los alumnos encuestados, el 14\% son de la carrera de Arquitectura, el 12\% de la carrera de Contador Público, el 16.5\% de la carrera de Ingeniería en Gestión Empresarial (IGE), el 7\% de Ingeniería en Sistemas Computacionales (ISC), el 9\% de Ingeniería Logística, el 14\% de Ingeniería Industrial, el 5\% de Ingeniería en Industrias Alimentarias, el 17\% de Ingeniería Mecatrónica y el 5.5\% de Licenciatura en Administración (LA).

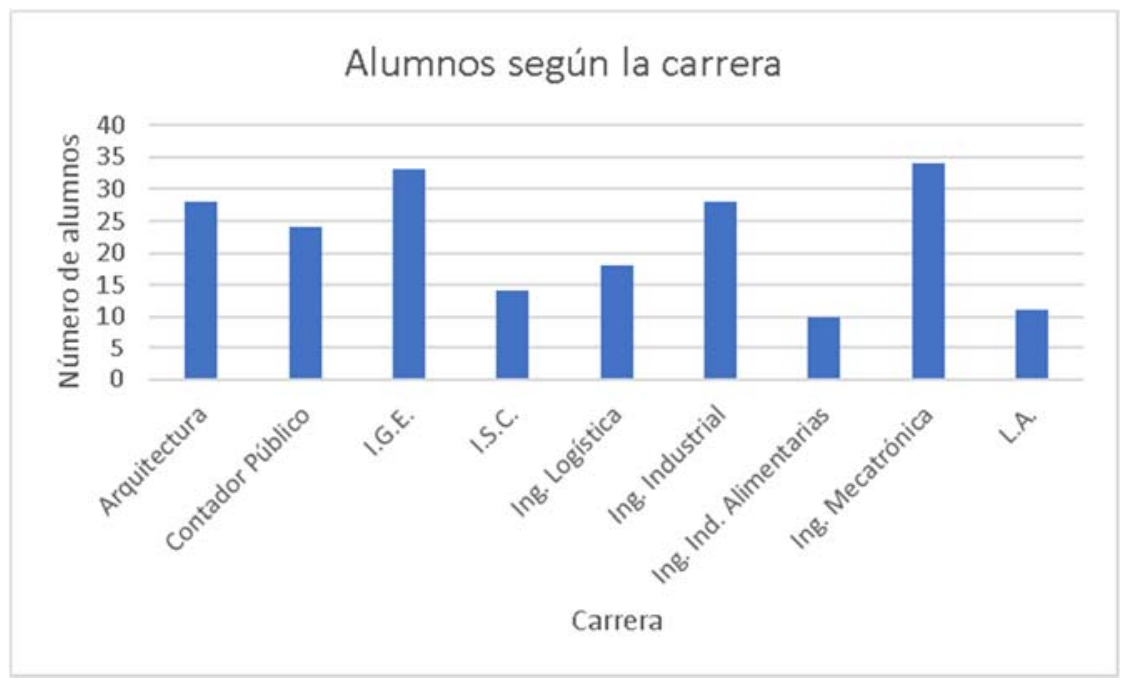

Fuente: Elaboración propia.

GUTIÉRREZ-ENRÍQUEZ E C. GUTIÉRREZ-ENRÍQUEZ K. I., JAQUES-SANDOVAL A., SANDOVAL-ACOSTA G., BUSTILLOSGONZÁLEZ J., RIVERA-QUEZADA J. L. 
EMPRENDIMIENTO SOCIAL EN LOS ESTUDIANTES DEL INSTITUTO TECNOLÓGICO DE CD. CUAUHTÉMOC.

De las 90 mujeres que contestaron la encuesta, se encontró que en promedio, el $11.11 \%$ de ellas está completamente de acuerdo en las 25 preguntas realizadas, mientras que el 23.33\% están en desacuerdo.

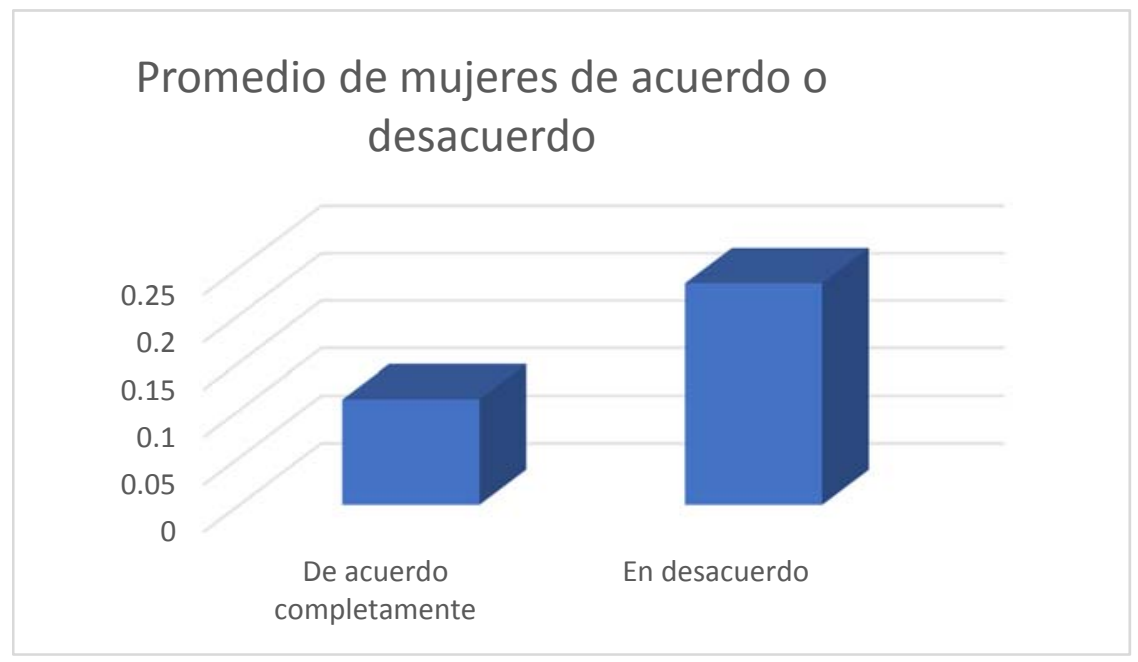

Figura 5. Media de las estudiantes mujeres.

Fuente: Elaboración propia.

De los 110 hombres que contestaron la encuesta, se encontró que en promedio, el 11.81\% de ellos está completamente de acuerdo en las 25 preguntas realizadas, mientras que el 20.90\% están en desacuerdo.

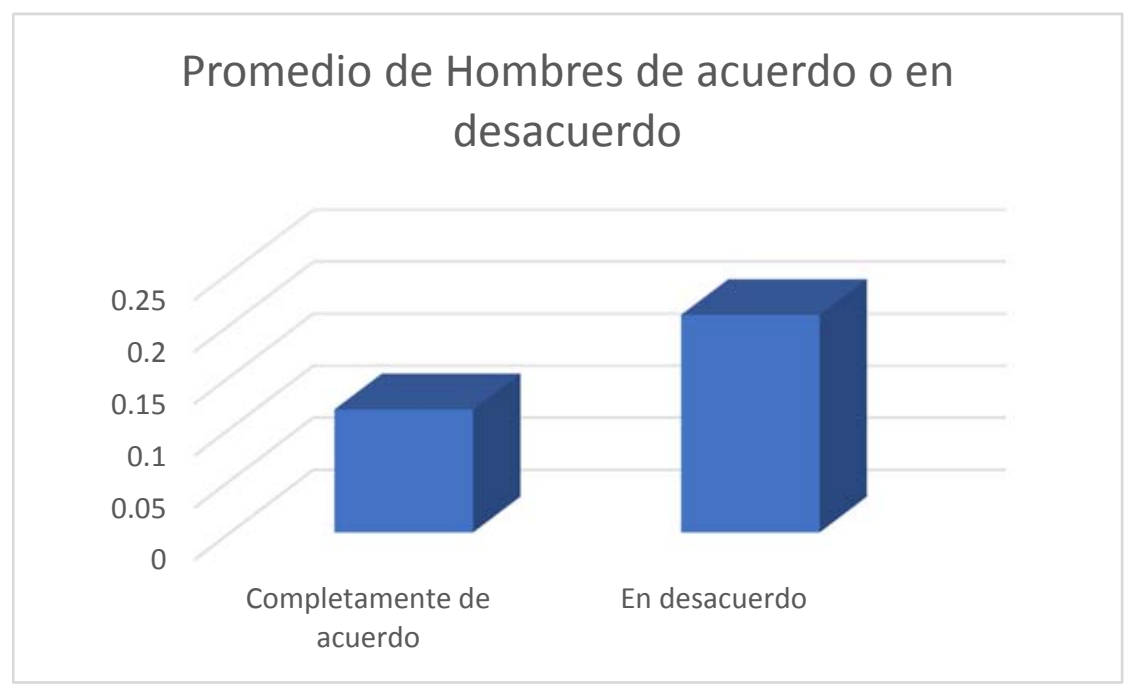

Figura 6. Media de los estudiantes hombres.

Fuente: Elaboración propia. 
EMPRENDIMIENTO SOCIAL EN LOS ESTUDIANTES DEL INSTITUTO TECNOLÓGICO DE CD. CUAUHTÉMOC.

De los 200 alumnos encuestados, encontramos que 67 son alumnos de primer semestre, 2 de segundo semestre, 61 de tercer semestre, 1 de cuarto semestre, 32 de quinto semestre, 27 de sétimo semestre y 10 de noveno semestre.

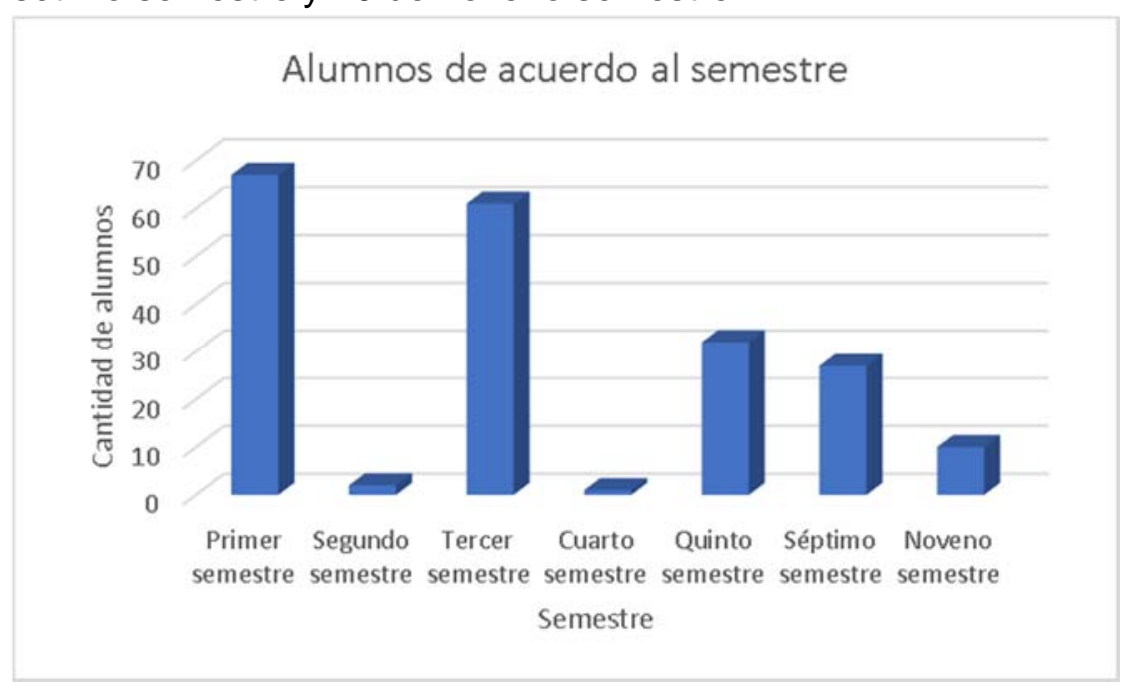

Figura 7. Alumnos por semestre.

Fuente: Elaboración propia.

\section{CONCLUSIONES}

Una vez obtenidos los resultados y analizando los datos se observa que el nivel de emprendimiento social es mediano, ya que menos del 30\% se esfuerza en ser el líder, en apoyar a sus compañeros o al realizar sus tareas, solo un 35\% manifiesta ser responsable de sus actos, sólo un 30\% de ellos busca involucrarse en proyectos nuevos. Es notorio, el nivel de conformismo y apatía que manifiestan los resultados.

El único aspecto en el que se presenta un nivel arriba del 50\%, es el de tomar riesgos, al salir de su comunidad a estudiar y al tomar materias difíciles.

El índice de conciencia social también se encuentra muy bajo, menos del 25\% de los alumnos encuestados manifiesta interés en los problemas de su comunidad, no apoyan a los indigentes ni a las personas de clase baja, un alto porcentaje, el $58 \%$ no ha donado sangre, por lo que se observa poca conciencia social.

Se observa una similitud en las formas de pensar y actuar de los estudiantes comparándolos por sexo genérico con lo cual se considera que él emprendimiento social y la conciencia social se GUTIÉRREZ-ENRÍQUEZ E C. GUTIÉRREZ-ENRÍQUEZ K. I., JAQUES-SANDOVAL A., SANDOVAL-ACOSTA G., BUSTILLOSGONZÁLEZ J., RIVERA-QUEZADA J. L. 
EMPRENDIMIENTO SOCIAL EN LOS ESTUDIANTES DEL INSTITUTO TECNOLÓGICO DE CD. CUAUHTÉMOC.

pudiera presentar de igual forma en hombres y mujeres. Se encuentra que se dan las mismas formas de pensar y actuar en los alumnos de Instituto Tecnológico por semestre y por licenciatura.

Las variables sociodemográficas no muestran diferencias o tendencias hacia el emprendimiento social, sólo el $11 \%$ de los encuestados presenta una tendencia favorable hacia las acciones de emprendimiento y conciencia social. El número de alumnos encuestados en los primeros semestres es mas alto, debido a que son grupos con mayor número de estudiantes, los semestres de quinto en delante tienen menor numero de integrantes.

Los estudiantes que participaron en el estudio no muestran las características que mencionan los autores, entre ellos UnLtd Spain (2019), quién considera que los emprendedores sociales son personas que se caracterizan por tener confianza en sí mismo, buscan trabajar en equipo, fuerte motivación, visión real del futuro, tienen capacidad de planificar y organizar y pueden mantener la comunicación de manera eficaz. Otras habilidades que poseen los emprendedores sociales son, la pasión por sus proyectos, liderazgo, son innovadores, bastante resilientes, pueden tomar decisiones en momentos difíciles, son inconformes con los problemas sociales, gran responsabilidad y compromiso social, toda vez que los resultados obtenidos son bajos, siendo el único con 55\% el correr riesgos de vivir en otra comunidad, esto quizá se debe a que algunos de ellos viven en comunidades alejadas del Instituto.

No se observa una tendencia al emprendimiento social o a la conciencia social, lo que lleva a concluir que los estudiantes del Instituto Tecnológico de $\mathrm{Cd}$. Cuauhtémoc, distan de tener características de emprendedores sociales.

\section{REFERENCIAS BIBLIOGRÁFICAS}

Carazo, J. (2020). Emprendimiento social, Economipedia, España. Recuperado de https://economipedia.com/definiciones/emprendimiento-social.html

Carranza, L. (2020). ¿Qué es el emprendimiento social?, blog emprende social, Perú. Recuperado de https://emprendesocial.com/sobre-nosotros/que-es-el-emprendimientosocial/ 
EMPRENDIMIENTO SOCIAL EN LOS ESTUDIANTES DEL INSTITUTO TECNOLÓGICO DE CD. CUAUHTÉMOC.

Del Cerro, (2015). Panorama: emprendimiento social en México, Entrepeneur, México. Recuperado de https://www.entrepreneur.com/article/268366

Disruptivo.tv. (2019). ¿Qué es emprendimiento social?, Disruptivo.tv, podcast. Recuperado de https://disruptivo.tv/que-es-emprendimiento-social/

Emprendimiento social. (2020). Emprendimiento como modo de vida, Venezuela. Recuperado de https://emprendimientosocial.info/emprendimiento/emprendimiento-como-modo-de-vida/

Fuentes, R., Limón, A. y Lázaro, M. (2018). Emprendimiento Social en México, Gestiopolis, México. Recuperado de https://www.gestiopolis.com/emprendimiento-social-en-mexico/

Fundación seres. (2017). ¿Qué es el emprendimiento social?, Fundación seres. Recuperado de https://www.fundacionseres.org/Paginas/Campus/BuenasPracticas.aspx?IDe=34

Instituto Nacional de Economía Social. (2018). Emprendedor social, el futuro del emprendimiento, Instituto Nacional de Economía Social, Gobierno de México. Recuperado de https://www.gob.mx/inaes/es/articulos/emprendedor-social-el-futuro-delemprendimiento?idiom=es

Ibarvo, V., Quijano, G. y Portillo, M. (2018). Índice para evaluar las diferencias del emprendimiento social entre los tecnológicos del TecNM de la Zona Norte y el Sureste de México, Investigación Instituto Tecnológico de Chihuahua, México.

Neetwork Business School. EOOD. (2020). Emprendimiento social. Definición y ejemplos, Neetwork Business School, Bulgaria. Recuperado de https://neetwork.com/emprendimiento-social-definicion-y-ejemplos/

Nuño, P. (2017). Emprendimiento social, Emprende Pyme.net, Valencia, España. Recuperado de https://www.emprendepyme.net/emprendimiento-social

Raffino M. (2018). Emprendimiento social. Concepto.de, Diccionario Online, Argentina. Recuperado de https://concepto.de/emprendimiento-social/

Rankia, S. L. (2019). ¿Qué es un emprendedor social?, Blog, Chile. Recuperado de https://www.rankia.cl/blog/ideas-emprendimiento-chile/4339381-que-emprendedor-social

Semana Educación: entrevista con Jaime Alberto Rincón, J. (2017). ¿Cómo entender el emprendimiento social?, Publicaciones semana S.A. Revista Online, Colombia. 
EMPRENDIMIENTO SOCIAL EN LOS ESTUDIANTES DEL INSTITUTO TECNOLÓGICO DE CD. CUAUHTÉMOC.

Recuperado de https://www.semana.com/educacion/articulo/que-es-emprendimientosocial/517782

UnLtd Spain. (2019). ¿Qué es un emprendedor social? Definición y características, Blog, España. Recuperado de https://www.unltdspain.org/2020/04/23/que-es-un-emprendedorsocial/\#: :text=El\%20objetivo\%20\%C3\%BAltimo\%20de\%20un,la\%20generaci\%C3\%B3n \%20de\%20beneficio\%20econ\%C3\%B3mico

Veredith, (2020). Emprendimiento social, parte de la vida actual. Recuperado de https://www.emprendices.co/emprendimiento-social-parte-de-la-vida-actual/

GUTIÉRREZ-ENRÍQUEZ E C. GUTIÉRREZ-ENRÍQUEZ K. I., JAQUES-SANDOVAL A., SANDOVAL-ACOSTA G., BUSTILLOSGONZÁLEZ J., RIVERA-QUEZADA J. L. 